\title{
New Approach to the Analysis of Raw Data from the Ocular Response Analyzer
}

\author{
Agnieszka Jóźwik ${ }^{a}$ Henryk Kasprzak ${ }^{a}$ Marta Kuczmab \\ a Department of Optics and Photonics, Wroclaw University of Technology, Wroclaw, and \\ ${ }^{b}$ Medical Clinic Oko-Laser, Krakow, Poland
}

\begin{abstract}
What Is It about?
We present a pilot study describing a new method of processing and analyzing raw data from the Ocular Response Analyzer (ORA) that can be used to describe and examine some new potential physical features of the anterior eye. Multiple measurements were made on 29 patients, distinguishing between the normal and the glaucoma group. The proposed new characteristics make it possible to differentiate the healthy reference group from the glaucoma patients.
\end{abstract}

\section{Keywords}

Eye - Anterior segment - Air puff tonometry - Ocular Response Analyzer - Eye biomechanics . Corneal applanation $\cdot$ Raw data

\begin{abstract}
Purpose: The aim of this research was to present a new approach for the potential characteristics of the anterior eye, based on numerical analysis of the raw data from multiple measurements on 1 eye captured with use of the Ocular Response Analyzer (ORA). Methods: The measurements were carried out 6 times on every eye, one by one, on 29 patients using the ORA. Dependencies between values of 6 applanation pressures $P_{1}$ and $P_{2}$ from 1 eye and the new proposed time-dependent parameters $\mathrm{sm}$ and smm, obtained from the raw data, were used to determine new characteristic features of the anterior eye. Results: Obtained values for multiple applanation pressures $\mathrm{P}_{1}$ and $\mathrm{P}_{2}$ and their average value $\mathrm{P}_{\mathrm{a}}$ from all measurements on 1 eye show strong linear dependencies with the new proposed time-dependent parameter smm. Conclusions: The new parameters based on slope coefficients $a_{1}$ and $a_{2}$, their difference $\Delta a$, and respective $y$-intercept values $b_{1}, b_{2}$, and $\Delta b$ show some characteristic stability for a multiply measured eye and can be used for its characterization. These parameters can be used for differentiation of the eyes with higher accuracy than those obtained from a single measurement. They are likely related to some biomechanical properties of the anterior eye.
\end{abstract}




\section{Introduction}

Intraocular pressure (IOP) is one of the parameters that are used in the diagnosis of glaucoma, in the monitoring of glaucoma progression, and in the evaluation of treatment effectiveness. The Ocular Response Analyzer (ORA; Reichert Technologies, Depew, NY, USA) is the first commercially available noncontact tonometer giving the possibility to measure, apart from Goldmann-correlated IOP (IOPg) and corneal compensated IOP (IOPcc), some specific biomechanical properties of the cornea, such as corneal hysteresis $(\mathrm{CH})$ and the corneal resistance factor (CRF) [1,2].

A single-pulse air stream from the pump bends the cornea. At the same time the corneal apex is illuminated by a laser diode, located out of axis of the measured eye. The pressure of the air puff is measured dynamically in the air jet, and the light intensity reflected from the corneal apex is synchronously detected by a photodiode, placed out of axis of the eye, symmetrically to the laser diode. The signal of the air puff pressure presents the pressure curve and consists of 400 measured points within $25 \mathrm{~ms}$. According to information given by the manufacturer, the air pump is switched off after the first corneal applanation; however, the air pressure still increases for another 3-4 ms. After having reached its maximum, the pressure starts decreasing to its initial value. The shape of the air pressure curve is basically similar to a Gaussian distribution, but more detailed analysis shows that the pressure curve is not symmetrical [3]. The second curve (applanation) given by the ORA also consists of 400 points measured within $25 \mathrm{~ms}$, but they represent the intensity of light reflected from the corneal center and registered by the photodiode. The applanation curve has 2 specific maximal values, where the corneal surface reaches 2 applanation states - during the inward and the outward corneal deformations. However, in many cases both applanation areas consist of a few local maxima, which usually complicates determination of the "best" applanation points. The ORA's software smoothens the applanation curve in order to get 2 clearer and explicitly distinct maxima, which define 2 applanation times $t_{1 \mathrm{a}}$ and $\mathrm{t}_{2 \mathrm{a}}$.

The applanation pressures $\mathrm{P}_{1}$ and $\mathrm{P}_{2}$ are estimated for the applanation times $\mathrm{t}_{1 \mathrm{a}}$ and $\mathrm{t}_{2 \mathrm{a}}$, from the air pressure curve [2]. The next 4 principal ORA parameters IOPg, IOPcc, $\mathrm{CH}$, and CRF are calculated from these values of applanation pressures $P_{1}$ and $P_{2}$. The ORA also gives an additional 37 parameters derived from the nonsmoothed applanation curve, which can be used for characterization of the applanation signal [4,5]. Some authors introduce new parameters or modify the parameters given by the ORA in order to make them more useful in ophthalmic diagnosis [6-8].

The waveform score (WS) has values ranging from 0 to 10 and describes the quality of the measurement. Various lower limits of acceptable values for WS were reported, giving reliable results of measurements. Usually these limits are suggested to be within the range of 4-7 [9-11]. However, according to our personal experience and results, the WS parameter describes not only the quality of measurements, but also the quality of corneal applanation and different corneal abnormalities. Low values of WS obtained for the same patient seem to give some valuable information about the corneal condition.

Repeated measurements of IOPg, IOPcc, $\mathrm{CH}$, and CRF for 1 patient give quite a significant distribution of results [12-14]. As proposed in the paper, more detailed analysis of these IOPg distributions and their dependencies on some parameters related to the geometry of both applanation and air pressure curves can manifest some interesting correlations. These dependencies can be used in determination of new parameters describing the properties of the cornea and the anterior eye.

This work presents a pilot study on new dependencies between parameters estimated from the raw data of both curves given by the ORA, and their potential application in determination of biomechanical properties of the anterior eye. 
Fig. 1. Data points for the region of the first applanation.

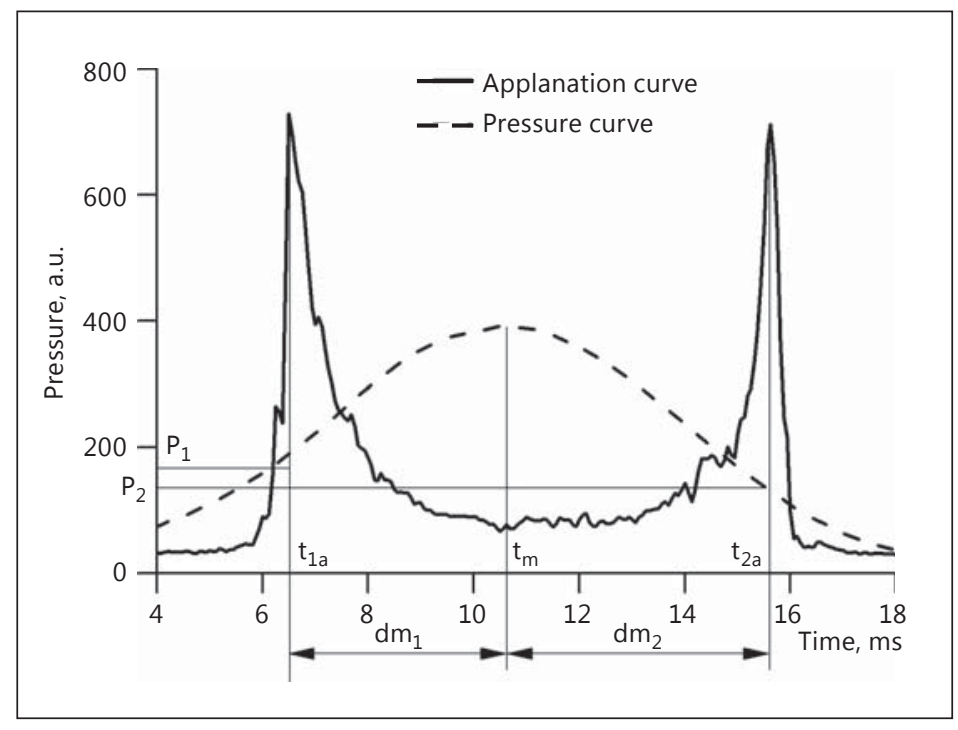

\section{Materials and Methods}

ORA measurements were repeatedly performed on 29 volunteers (aged 25-78 years, mean \pm SD $51.4 \pm 18.5$ years) between January and March 2013. Ten patients (aged 25-39 years, mean \pm SD $32.2 \pm 5.0$ years) without identification of glaucoma were classified as the normal group, while 19 subjects (aged $32-78$ years, mean \pm SD $61.6 \pm 14.3$ years) were classified as the glaucomatous group. The ORA gives 4 results of the measured parameter, which are usually averaged by the examiner. In the current study a different approach is proposed: more measurements were performed on each patient, and some characteristic features of these measurements were taken for further consideration and analysis. In order not to cause discomfort to the subjects, 6 measurements were performed on each examined eye. However, in order to test the repeatability of examined features, the eyes of 9 subjects from the healthy group were measured 15 times. All measurements were performed within 1 day, with at least 15-min intervals between the consecutive measurements. All patients were fully informed about the details of these noninvasive procedures as well as the purpose and possible consequences of the study. Conscious agreement was obtained from the subjects. The research followed the tenets of the Declaration of Helsinki and was approved by the Ethics Committee of the Medical University of Wroclaw (KB 503/2011).

The results were repeatable even for lower values of WS that are usually eliminated [9-11]. However, WS measurements below 2 were rejected due to difficulty of numerical analysis, caused by significantly increasing dispersal of calculated parameters. The raw data points of the air pressure and applanation curves, exported directly from the instrument, were numerically processed in a MATLAB environment (Math Works, Inc., Natick, MA, USA, version 2011a).

In order to find the optimal value of both the first $t_{1 a}$ and the second $t_{2 a}$ applanation times from the raw data, 5 different methods were used to estimate the timing of both applanations.

The first 2 methods were based on calculation of the center of mass (centroid) of the 2 areas of the applanation curve close to the applanation region. The applanation region was always selected as the region where the values of the applanation curve exceeded $70 \%$ of the maximal peak for the respective applanation. The data points of the first applanation region and their smoothing are shown in Figure 1. In the first method, the centroid (representing 
Fig. 2. Considered parameters describing air pressure and applanation curves.

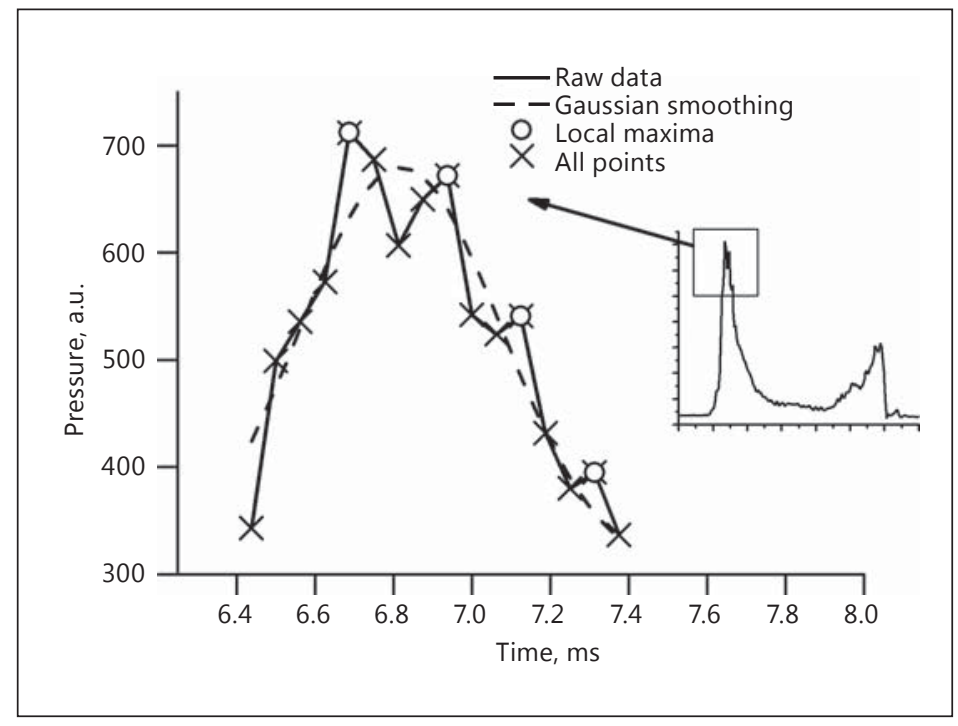

times $t_{1 a}$ or $t_{2 a}$ ) was calculated only from the local maxima in the established applanation region, while all the data points from this region were taken into account for centroid calculation in the second method. The third and fourth methods used the same data points as the first and the second method, but instead of centroids, the centers of inertia were calculated. The fifth method estimated the applanation time from the maximum of the smoothed applanation curve. The original, raw applanation curve was smoothed by means of a Gaussian filter with the window size equal to 17 points. The size of the window was selected from the highest correlation, presented in the next section. The respective values of the applanation pressures $\mathrm{P}_{1}$ and $\mathrm{P}_{2}$ were calculated for each measurement from applanation times $\mathrm{t}_{1 \mathrm{a}}$ and $\mathrm{t}_{2 \mathrm{a}}$, obtained for all 5 methods.

While testing and estimating a number of different dependencies between both the applanation air pressures $\mathrm{P}_{1}, \mathrm{P}_{2}$, or their arithmetic average $\mathrm{P}_{\mathrm{a}}$ and time-related parameters of the air pressure and applanation curves, some interesting correlations were observed. The highest, unexpected characteristic correlations for 1 patient were observed between pressures $\mathrm{P}_{1}, \mathrm{P}_{2}$, or $\mathrm{P}_{\mathrm{a}}$, and two time-related parameters of both ORA curves, defined as

$$
\begin{aligned}
& s m=\frac{t_{m}}{d m}, \\
& s m m=\frac{t_{m}}{\sqrt{d m_{1} \times d m_{2}}}-1,
\end{aligned}
$$

the time periods $t_{m}, d m_{1}$, and $d m_{2}$ being shown in Figure 2. The parameter $t_{m}$ is the time of maximum air pressure, and $\mathrm{dm}$ is the time between two applanations (sum of $\mathrm{dm}_{1}$ and $\mathrm{dm}_{2}$ ).

The relationships between the values $\mathrm{P}_{1}, \mathrm{P}_{2}$, or $\mathrm{P}_{\mathrm{a}}$ and smm or sm show strong linear dependency for each eye under examination. The following parameters were calculated for all dependencies: slope a, $y$-intercept $\mathrm{b}$ and the Pearson correlation coefficient $R$. The calculations were performed for all the 5 methods of applanation time determination. Statistical analysis of linear dependency was performed using 2-tailed test significance of determination. A $p$ value $<0.05$ indicates a statistically significant difference between 2 parameters. 
(c) 2016 The Author(s). Published by S. Karger AG, Basel www.karger.com/bmh

Jóźwik et al.: New Approach to the Analysis of Raw Data from the Ocular Response Analyzer

Table 1. Values of Pearson correlation coefficients $R$ for 29 patients for dependencies between pressures $P_{1}$, $\mathrm{P}_{2}$, or $\mathrm{P}_{\mathrm{a}}$ and sm or smm for 29 patients

\begin{tabular}{|c|c|c|c|c|c|c|}
\hline Patient No. & $\mathrm{P}_{1}(\mathrm{sm})$ & $\mathrm{P}_{2}(\mathrm{sm})$ & $\mathrm{P}_{\mathrm{a}}(\mathrm{sm})$ & $\mathrm{P}_{1}(\mathrm{smm})$ & $\mathrm{P}_{2}(\mathrm{smm})$ & $\mathrm{P}_{\mathrm{a}}(\mathrm{smm})$ \\
\hline 1 & 0.875 & 0.971 & 0.992 & 0.885 & 0.962 & 0.992 \\
\hline 2 & 0.796 & 0.986 & 0.969 & 0.823 & 0.978 & 0.977 \\
\hline 3 & 0.793 & 0.920 & 0.979 & 0.787 & 0.921 & 0.977 \\
\hline 4 & 0.998 & 0.989 & 0.997 & 0.998 & 0.987 & 0.996 \\
\hline 5 & 0.914 & 0.967 & 0.975 & 0.921 & 0.966 & 0.977 \\
\hline 6 & 0.775 & 0.993 & 0.968 & 0.890 & 0.986 & 0.992 \\
\hline 7 & 0.978 & 0.978 & 0.993 & 0.964 & 0.979 & 0.994 \\
\hline 8 & 0.974 & 0.986 & 0.994 & 0.986 & 0.965 & 0.989 \\
\hline 9 & 0.915 & 0.864 & 0.995 & 0.894 & 0.883 & 0.995 \\
\hline 10 & 0.895 & 0.979 & 0.968 & 0.906 & 0.975 & 0.972 \\
\hline 11 & 0.997 & 0.977 & 0.995 & 0.995 & 0.967 & 0.991 \\
\hline 12 & 0.977 & 0.927 & 0.974 & 0.984 & 0.922 & 0.975 \\
\hline 13 & 0.971 & 0.958 & 0.999 & 0.969 & 0.960 & 0.997 \\
\hline 14 & 0.888 & 0.750 & 0.997 & 0.899 & 0.654 & 0.989 \\
\hline 15 & 0.959 & 0.887 & 0.989 & 0.954 & 0.627 & 0.951 \\
\hline 16 & 0.877 & 0.540 & 0.988 & 0.908 & 0.608 & 0.970 \\
\hline 17 & 0.720 & 0.903 & 0.958 & 0.875 & 0.933 & 0.974 \\
\hline 18 & 0.574 & 0.757 & 0.921 & 0.425 & 0.815 & 0.932 \\
\hline 19 & 0.997 & 0.998 & 0.999 & 0.999 & 0.998 & 0.999 \\
\hline 20 & 0.865 & 0.951 & 0.988 & 0.957 & 0.888 & 0.962 \\
\hline 21 & 0.838 & 0.854 & 0.977 & 0.889 & 0.904 & 0.987 \\
\hline 22 & 0.907 & 0.972 & 0.994 & 0.880 & 0.936 & 0.995 \\
\hline 23 & 0.656 & 0.970 & 0.991 & 0.784 & 0.732 & 0.967 \\
\hline 24 & 0.942 & 0.940 & 0.974 & 0.768 & 0.892 & 0.916 \\
\hline 25 & 0.975 & 0.984 & 0.992 & 0.984 & 0.970 & 0.992 \\
\hline 26 & 0.843 & 0.946 & 0.970 & 0.851 & 0.953 & 0.977 \\
\hline 27 & 0.987 & 0.997 & 0.999 & 0.988 & 0.998 & 0.998 \\
\hline 28 & 0.975 & 0.937 & 0.981 & 0.963 & 0.947 & 0.984 \\
\hline 29 & 0.892 & 0.909 & 0.990 & 0.912 & 0.897 & 0.999 \\
\hline
\end{tabular}

\section{Results}

Linear dependencies with high correlation coefficients and $p$ values $<0.05$ between pressures of applanation $\mathrm{P}_{1}, \mathrm{P}_{2}, \mathrm{P}_{\mathrm{a}}$ and parameters sm or smm were obtained for all 29 patients with repetition of 6 measurements. Pearson correlation coefficients are presented in Table 1. The results show that the difference between calculations of parameters from 15 and 6 measurements was not statistically significant $(p>0.05)$. Numerical analysis showed that correlations between both pressures $\mathrm{P}_{1}$ or $\mathrm{P}_{2}$ and the smm parameter were slightly larger than the correlations between both pressures and sm for the majority of measurements. The differences in the obtained correlations calculated for the 5 methods of the estimation of applanation timing described above were small, and there was no statistical difference (Wilcoxon test) between them ( $p>0.05$ ). Significant differences were obtained for signals with WS $<2$, but these data were not used for further analysis. The first method of calculation of both applanation times with the centroid of the local maxima was chosen for further consideration.

Some exemplary results obtained for 2 patients are shown in Figure 3. High correlations between $\mathrm{P}_{1}, \mathrm{P}_{2}$, and smm can be observed. However, the highest correlation was always between $\mathrm{P}_{\mathrm{a}}$ and smm $(R>0.95)$. Such a very high correlation is rarely observed in measure- 
Fig. 3. Dependencies between respective applanation pressure values $\left(\mathrm{P}_{1}\right.$, highest line; $\mathrm{P}_{2}$, lowest line; $\mathrm{P}_{\mathrm{a}}$, central line) and smm parameter for 2 exemplary patients.

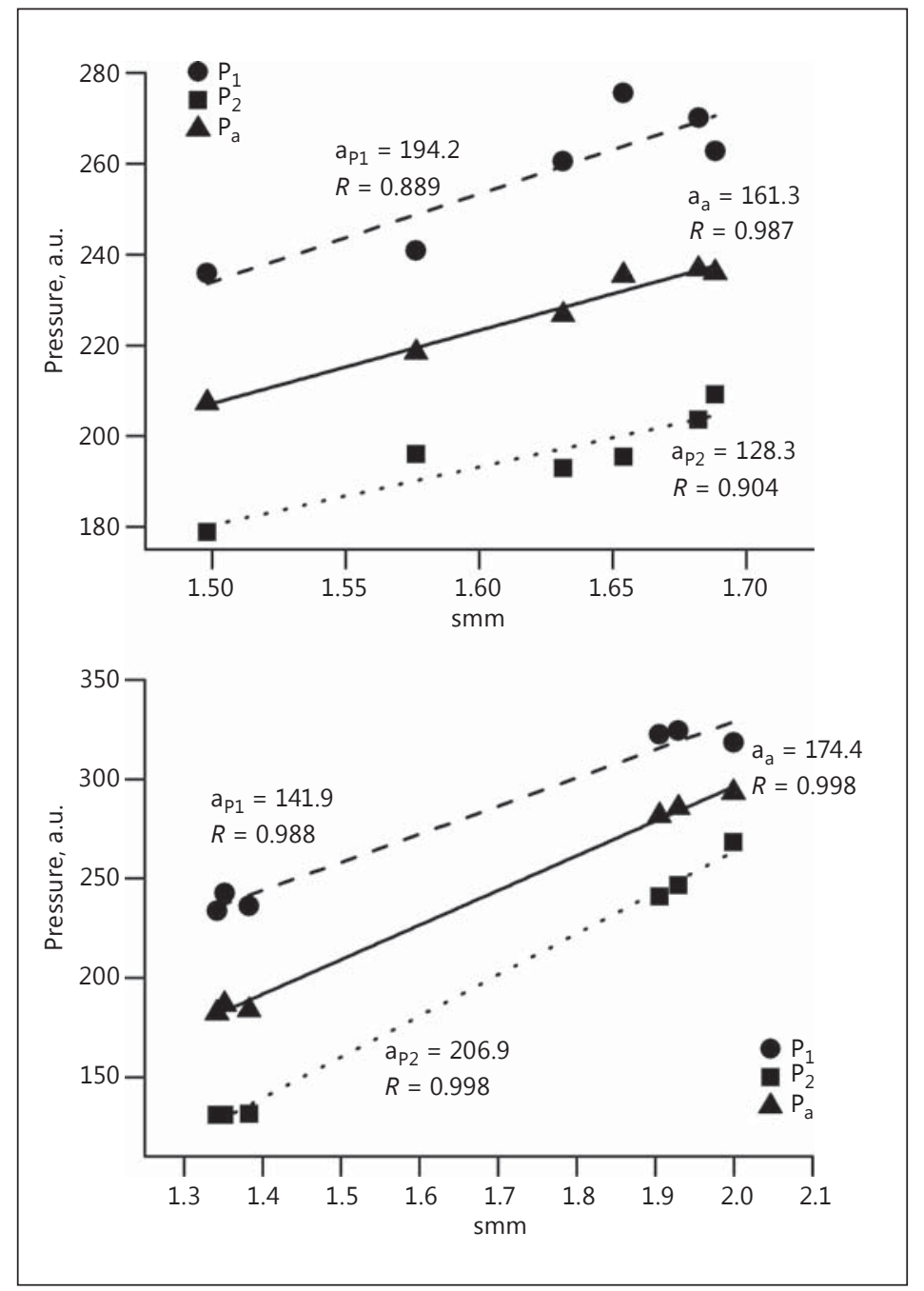

ments of biological parameters. An additional interesting feature is that the dispersion of data points for $\mathrm{P}_{\mathrm{a}}$ from the straight line is lower than those for both the $\mathrm{P}_{1}$ and $\mathrm{P}_{2}$ lines in all cases; the respective correlation coefficient was close to 1 . It is worth noticing that such high correlations appeared even for relatively low values of WS. Slopes $\mathrm{a}_{\mathrm{P} 1}, \mathrm{a}_{\mathrm{P} 2}$, and $\mathrm{a}_{\mathrm{a}}$ and the respective $y$-intercept values $b_{\mathrm{P} 1}, b_{\mathrm{P} 2}$, and $b_{\mathrm{a}}$ were calculated for all patients, for the 3 dependencies presented in Figure 3. Differences between the slopes and the y-intercept values of both curves representing pressures $\mathrm{P}_{1}$ and $\mathrm{P}_{2}$ were calculated as follows:

$$
\begin{aligned}
& \Delta a=a_{\mathrm{P} 1}-a_{\mathrm{P} 2}, \\
& \Delta b=b_{\mathrm{P} 1}-b_{\mathrm{P} 2} .
\end{aligned}
$$

It seems to be expected that according to hysteresis properties, both the $\mathrm{P}_{1}$ and $\mathrm{P}_{2}$ lines should be more or less parallel. However, our analysis showed that for some patients these 2 lines were converging and for the other diverging, giving positive or negative differences $\Delta \mathrm{a}$. The values of $\Delta$ a were distributed almost symmetrically with relation to zero. The distribution of $\Delta$ a values was close to a normal distribution at the level of significance $\alpha=0.05$ (Shapiro-Wilk test). For more than 6 measurements on 1 eye, the correlations between the parameters were comparable. However, by increasing the number of repeated measurements on 1 eye, the examination becomes more uncomfortable for the patient. 
Fig. 4. Dependencies between $\Delta \mathrm{a}$ and $\mathrm{a}_{\mathrm{p} 1}$ (dashed line, circles) as well as $\mathrm{a}_{\mathrm{P} 2}$ (solid line, crosses) for 29 patients.
(C) 2016 The Author(s). Published by S. Karger AG, Basel www.karger.com/bmh

Jóźwik et al.: New Approach to the Analysis of Raw Data from the Ocular Response Analyzer

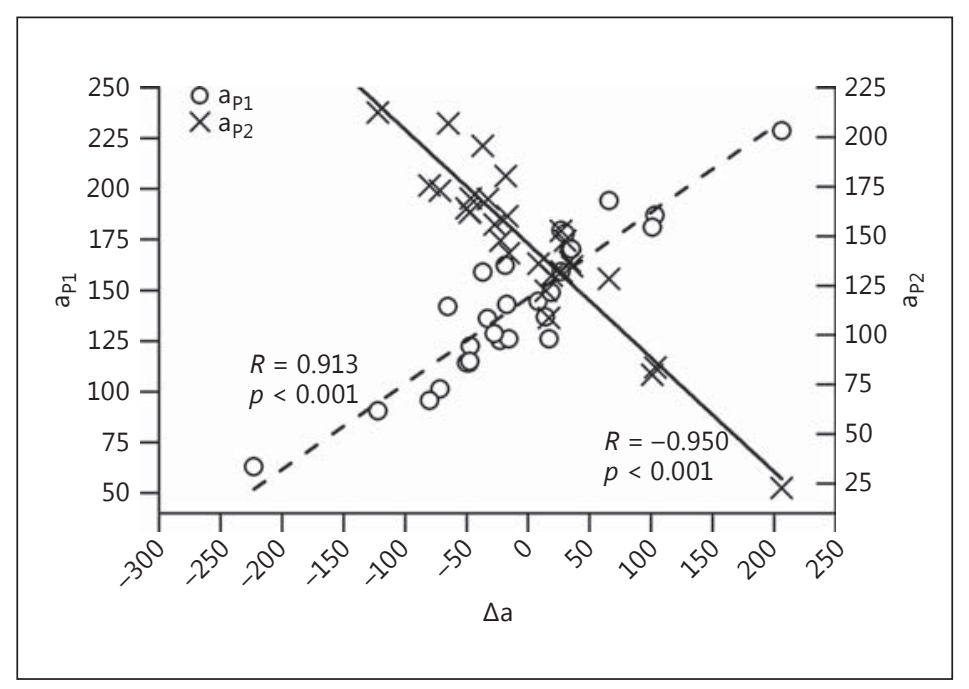

It was noticeable that $\Delta$ a was strongly dependent on the values of $\mathrm{a}_{\mathrm{P} 1}(R=0.913, p<$ $0.001)$ and $\mathrm{a}_{\mathrm{P} 2}(R=-0.950, p<0.001)$. Figure 4 presents the values of slopes $\mathrm{a}_{\mathrm{P} 1}$ and $\mathrm{a}_{\mathrm{P} 2}$ plotted as a function of the slope differences $\Delta \mathrm{a}$, as well as a high correlation between $\Delta \mathrm{a}$ and y-intercepts $\mathrm{b}_{\mathrm{P} 1}(R=0.893)$ and $\mathrm{b}_{\mathrm{P} 2}(R=0.957)$. All dependencies were statistically significant. There was no significant correlation between $\Delta \mathrm{a}$ and $\mathrm{a}_{\mathrm{a}}(R=0.375)$ and $\mathrm{b}_{\mathrm{a}}(R=0.458)$. The analysis of the $\Delta \mathrm{b}$ parameter also manifested a very high correlation to the parameters: $\mathrm{a}_{\mathrm{P} 1}(R=0.910), \mathrm{a}_{\mathrm{P} 2}(R=0.929), \mathrm{b}_{\mathrm{P} 1}(R=0.922)$, and $\mathrm{b}_{\mathrm{P} 2}(R=0.958)$. The Shapiro-Wilk test showed that there is no reason to reject the hypothesis of a lack of normality of the distribution of $\Delta \mathrm{b}$ values. Similar to the case of the slope differences $\Delta \mathrm{a}$, there were low Pearson correlation coefficients between $\Delta \mathrm{b}$ and $\mathrm{a}_{\mathrm{a}}(R=0.347)$ or $\mathrm{b}_{\mathrm{a}}(R=0.426)$ values. It is worth noticing that $\Delta \mathrm{a}$ and $\Delta \mathrm{b}$ were better correlated to the parameters related to the second applanation $\left(\mathrm{a}_{\mathrm{P} 2}\right.$ and $\left.\mathrm{b}_{\mathrm{P} 2}\right)$ than to the respective parameters of the first applanation $\left(\mathrm{a}_{\mathrm{P} 1}\right.$ and $\left.\mathrm{b}_{\mathrm{P} 1}\right)$.

The values of the slope $\mathrm{a}_{\mathrm{p} 1}$ showed lack of correlation to the slope of average pressures $\mathrm{a}_{\mathrm{a}}(R=0.035, p=0.86)$, while the values of the slope $\mathrm{a}_{\mathrm{P} 2}$ were moderately correlated to $\mathrm{a}_{\mathrm{a}}$ $(R=0.646, p<0.001)$. Similar results were observed for $\mathrm{y}$-intercept values $\mathrm{b}_{\mathrm{P} 1}$ versus $\mathrm{b}_{\mathrm{a}}(R=$ $-0.043, p=0.82)$ and $\mathrm{b}_{\mathrm{P} 2}$ versus $\mathrm{b}_{\mathrm{a}}(R=0.668, p<0.001)$.

Further analysis showed that the values $\Delta$ a were neither correlated to the average values of $\mathrm{CH}(R<0.1)$ nor to the values of IOPg $(R<0.1)$. It is likely that the value $\Delta$ a is related to some other biomechanical features of the anterior eye.

Figure 5 presents the high correlation between $\Delta \mathrm{a}$ and $\mathrm{b}_{\mathrm{P} 1}(R=-0.893, p<0.001)$. One could notice that there was a higher concentration of results for healthy subjects in the center of the graph, while the results obtained for glaucomatous patients manifested larger dispersion. However, in some cases the results for the glaucomatous patients were also localized in the central part of the graph.

Interesting results can be observed in Figure 6, which presents the values of $b_{a}$ plotted against the values of $\mathrm{a}_{\mathrm{a}}$. In general, all results can be approximated by a linear function (continuous line) with a relatively high correlation $(R=-0.884, p<0.001)$. If one separates the healthy group (circles) from the glaucomatous patients (crosses), it can be observed that both groups can be approximated by 2 different lines with significantly higher correlations ( $R=-0.978$ for normal subjects and $R=-0.981$ for glaucomatous patients). Linear approximation can be written as

$$
b_{\mathrm{a}}=-1.42 \times a_{\mathrm{a}}+176
$$


Fig. 5. Dependencies between $\Delta \mathrm{a}$ and $b_{\mathrm{P} 1}$ for normal (circles) and glaucoma (crosses) patients.
(C) 2016 The Author(s). Published by S. Karger AG, Basel www.karger.com/bmh

Jóźwik et al.: New Approach to the Analysis of Raw Data from the Ocular Response Analyzer

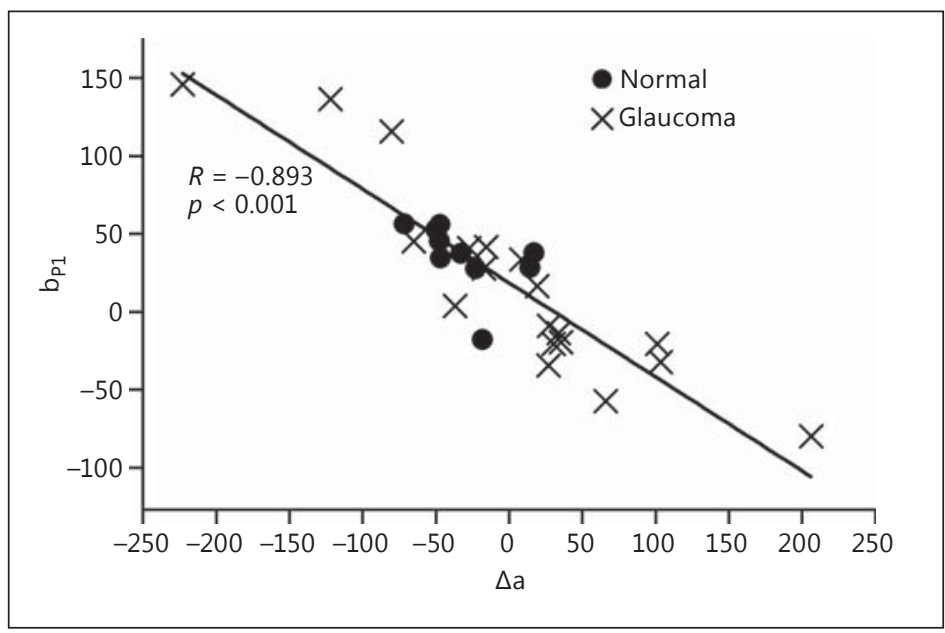

Fig. 6. Variability of $y$-intercept $b_{a}$ parameter versus average slope $\mathrm{a}_{\mathrm{a}}$, separated into 2 groups.

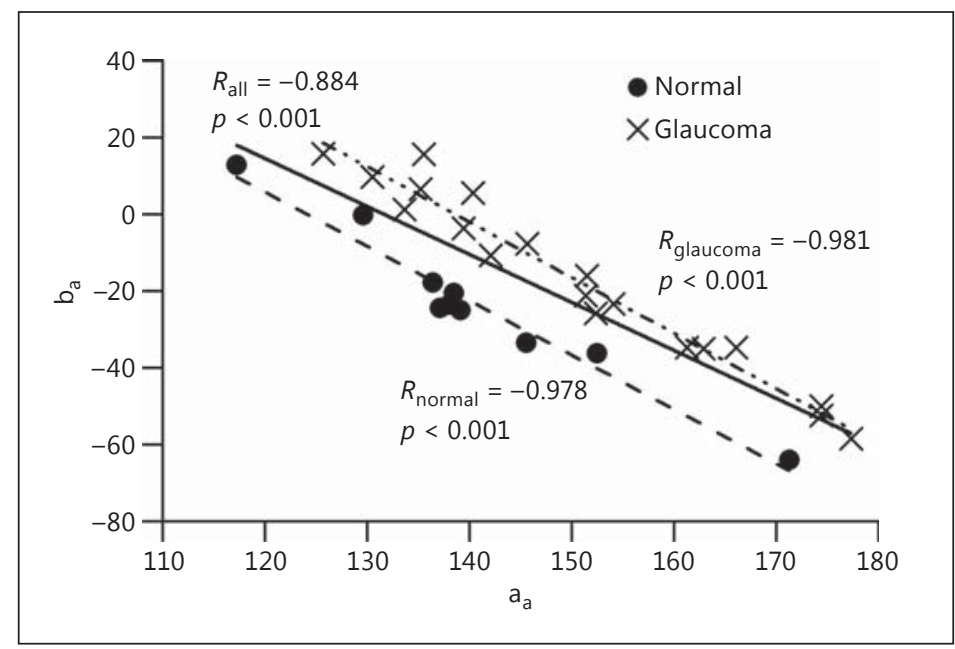

for the normal group, and

$$
b_{\mathrm{a}}=-1.44 \times a_{\mathrm{a}}+200
$$

for the glaucomatous patients. Statistical analysis showed significant differences in the parameters of these lines.

The difference between the 2 groups was particularly visible in the 3D graph, where the respective values of $\Delta$ a were taken into account and plotted as the third axis (Fig. 7). The respective points representing the 2 groups of patients were located in 2 different planes.

The planar approximation on a 3 graph for the glaucoma patients is represented by the equation:

$$
b_{\mathrm{a}}=0.141 \times \Delta a-1.33 \times a_{\mathrm{a}}+168,
$$

with multiple correlation coefficient $R=0.996$ while for the healthy subjects:

$$
b_{\mathrm{a}}=-0.018 \times \Delta a-1.50 \times a_{\mathrm{a}}+209,
$$

with respective multiple correlation coefficient $R=0.984$. 


\section{क्ष}

Fig. 7. 3D graph of $b_{a}$ values versus slope difference $\Delta \mathrm{a}$ and slope $\mathrm{a}_{\mathrm{a}}$, with respective planar approximations for 2 groups of patient with their projections on 3 respective planes.

Fig. 8. Results of measurements and linear regression for dependency between IOPg obtained from the ORA and $\mathrm{P}_{\mathrm{a}}$ (black line) as well as the line defined by equation 10 (gray line).
Biomed Hub 2016;1:452124 (DOI: 10.1159/000452124) www.karger.com/bmh

Jóźwik et al.: New Approach to the Analysis of Raw Data from the Ocular Response Analyzer
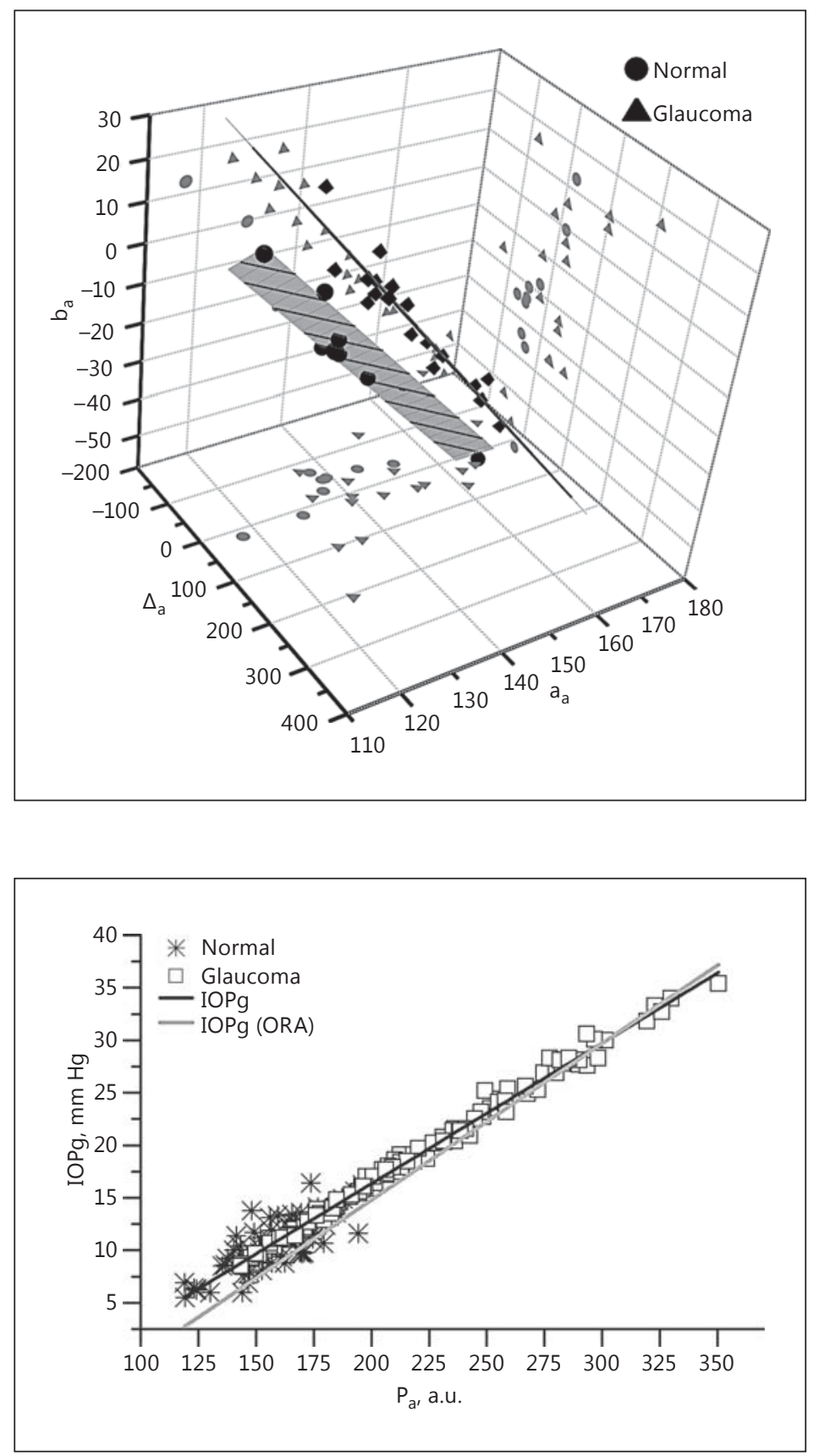

In order to examine the correlation between the average value of both applanation pressures $\mathrm{P}_{\mathrm{a}}$ obtained from raw data and the value of IOPg given by the ORA, all measurements for 29 patients $(29 \times 6)$ were taken into account. Figure 8 shows a very high correlation between the examined values $(R=0.986, p<0.001)$. Estimated linear approximation is given by the equation

$$
I O P g=P_{\mathrm{a}} \times 0.134-10.5 .
$$

However, the equation given by the ORA's manufacturer [1] for the same dependency differs from the one above: 
Both lines are marked in Figure 8. One can see that the results for the healthy subjects (crosses) characterize higher dispersion in comparison to those of glaucoma patients (squares), but both of them are not located on the line defined by equation 9 .

\section{Discussion}

This paper proposes a new method of processing and analysis of the raw data from the ORA instrument. According to the ORA's software suggestion, usually 1-4 measurements are taken on 1 eye, and the results for the highest values of WS parameter are the final results of the measurement. However, consecutive repetition of the measurements on the same eye can give quite a wide distribution of obtained results. Interpretation of this distribution is still not clear. It is very likely that the anterior eye changes its physical features during quasi-periodical IOP variations due to blood pulsation [15]. Usually, the values of IOPg given by the ORA are averaged, and its mean value is treated as the closest to the real one. It determines an optimal and the simplest approach. However, such an approach should lead to 2 important questions: (1) are these distributed 4 magnitudes correlated to some other characteristics of the measurements? (2) Is there any additional useful information hidden in these distributed results?

In order to examine the correlation between some new parameters obtained from the raw data of different measurements on 1 eye, we propose to perform at least 6 consecutive measurements. As was shown in this paper, the high correlations between values of both applanation pressures $\mathrm{P}_{1}$ and $\mathrm{P}_{2}$ and the respective time-dependent parameter smm make it possible to define some new parameters. These parameters are the slopes $\mathrm{a}_{\mathrm{P} 1}, \mathrm{a}_{\mathrm{P} 2}$, and $\mathrm{a}_{\mathrm{Pa}}$ of 3 lines, the fitting applanation pressure values $\mathrm{P}_{1}, \mathrm{P}_{2}$, and their arithmetic average $\mathrm{P}_{\mathrm{a}}$ versus smm, and the respective difference $\Delta a$ as well as the $y$-interceptions $\mathrm{b}_{\mathrm{P} 1}, \mathrm{~b}_{\mathrm{P} 2}$, and $\mathrm{b}_{\mathrm{Pa}}$ and the difference $\Delta \mathrm{b}$. Interestingly, the very high correlation between $\mathrm{P}_{\mathrm{a}}$ and smm is always higher than both the high correlations between $\mathrm{P}_{1}, \mathrm{P}_{2}$, and smm. On the other hand, the correlations between newly introduced parameters and $\mathrm{a}_{\mathrm{P} 2}$ are almost always higher than those for the $\mathrm{a}_{\mathrm{P} 1}$ and $\mathrm{a}_{\mathrm{Pa}}$ parameters. Unexpectedly, lines fitting dependencies $\mathrm{P}_{1}$ and $\mathrm{P}_{2}$ versus smm can be parallel $(\Delta \mathrm{a} \cong 0)$, divergent $(\Delta \mathrm{a}>0)$ or convergent $(\Delta \mathrm{a}<0)$. It is likely that the value $\Delta \mathrm{a}$ describes some new biomechanical feature of the anterior part of the eye. However, there is no correlation between $\Delta \mathrm{a}$ and the average value of $\mathrm{CH}$ for measured patients. Similarly, like in Fung [16] who introduced two independent constants $A$ and $\alpha$ for description of nonlinearity of the tissue elasticity, its viscoelasticity can be also described by 2 or more independent constants, not only by a single $\mathrm{CH}$ parameter. The proposed parameter $\Delta \mathrm{a}$ can be likely treated as an independent parameter describing corneal viscoelasticity.

The analysis of the proposed parameters for the 2 groups of patients showed that, by means of these parameters, it is possible to differentiate the group of healthy subjects from the group of glaucoma patients.

The statistical analysis carried out in this study showed that the obtained linear approximation of averaged applanation pressures $P_{1}$ and $P_{2}$, calculated as $P_{a}$ with IOPg values given by the ORA, is described by different equations (equations 9 and 10) than the one given by the ORA's producer.

The new dependencies were obtained from the raw data given directly by the ORA. Access to the raw ORA data enables a new approach to quantitative analysis of the corneal biomechanics and determination of the new parameters, in order to describe and examine some new physical features of the anterior eye. Such an approach may broaden the usefulness of the ORA instrument in ophthalmic diagnosis. 


\section{Acknowledgments}

The authors are grateful for the medical assistance provided by Patrycja KrzyzanowskaBerkowska, MD from the Department of Ophthalmology, Wroclaw Medical University, Poland.

\section{Disclosure Statement}

The authors report no conflicts of interest. They alone are responsible for the content and writing of the paper.

\section{References}

$>1$ Luce DA: Determining in vivo biomechanical properties of the cornea with an ocular response analyzer. J Cataract Refract Surg 2005;31:156-162.

-2 Reinstein DZ, Gobbe M, Archer TJ: Ocular biomechanics: measurement parameters and terminology. J Refract Surg 2011;27:396-397.

-3 Kasprzak H, Mazur E, Widlicka M: Measurement and analysis of the air pressure curve on the rigid lenses by use of Ocular Response Analyzer. Acta Bioeng Biomech 2014;16:117-121.

4 Luz A, Fontes BM, Lopes B, Ramos I, Schor P, Ambrósio R: ORA waveform-derived biomechanical parameters to distinguish normal from keratoconic eyes. Arq Bras Oftalmol 2013;76:111-117.

5 Lam AKC, Chen D, Tse J: The usefulness of waveform score from the ocular response analyzer. Optom Vis Sci 2010;87:195-199.

6 Touboul D, Benard A, Mahmoud AM, Gallois A, Colin J, Roberts CJ: Early biomechanical keratoconus pattern measured with an Ocular Response Analyzer: curve analysis. J Cataract Refract Surg 2011;37:2144-2150.

7 Kotecha A, Elsheikh A, Roberts CR, Zhu H, Garway-Heath DF: Corneal thickness- and age-related biomechanical properties of the cornea measured with the Ocular Response Analyzer. Invest Ophthalmol Vis Sci 2006; 47:5337-5347.

8 Lau W, Pye D: A clinical description of Ocular Response Analyzer measurements. Invest Ophthalmol Vis Sci 2011;52:2911-2916.

-9 El-Malah MA: Evaluation of corneal biomechanics using ocular response analyzer for normal and primary open angle glaucoma eyes. J Egypt Ophthalmol Soc 2013;106:249-252.

10 Kotecha A, Russell RA, Sinapis A, Pourjavan S, Sinapis D, Garway-Heath DF: Biomechanical parameters of the cornea measured with the Ocular Response Analyzer in normal eyes. BMC Ophthalmol 2014;14:11.

-11 Vantomme M, Pourjavan S, Detry-Morel M: The range of the waveform score of the ocular response analyzer (ORA) in healthy subjects. Bull Soc Belge Ophtalmol 2013;322:91-97.

-12 Rogowska ME, Iskander DR, Kasprzak HT: Assessing subject-related variations of the Ocular Response Analyzer parameter calculation. Clin Exp Optom 2015;98:348-352.

13 Zareei A, Razeghinejad MR, Nowroozzadeh MH, Mehrabi Y, Aghazadeh-Amiri M: Intraocular pressure measurement by three different tonometers in primary congenital glaucoma. J Ophthalmic Vis Res 2015;10: 43-48.

14 Bayoumi NHL, Bessa AS, El Massry AAK: Ocular Response Analyzer and Goldmann applanation tonometry: a comparative study of findings. J Glaucoma 2010;19:627-631.

15 Asejczyk-Widlicka M, Krzyżanowska-Berkowska P, Kowalska M, Iskander DR: Clinical utility of spectral analysis of intraocular pressure pulse wave. BMC Ophthalmol 2014;14:30.

16 Fung YC: Biomechanics: Mechanical Properties of Living Tissues. New York, Springer, 1993. 